Michiko Mizoguchi · Takashi Tamura • Akiko Yamaki

Eiji Higashihara Y Yoshiko Shimizu

\title{
Mutations of the PKD1 gene among Japanese autosomal dominant polycystic kidney disease patients, including one heterozygous mutation identified in members of the same family
}

Received: April 21, 2001 / Accepted: June 11, 2001

\begin{abstract}
More than 80 mutations of the $P K D 1$ gene have been reported, mostly in patients from Western Europe. New techniques are being used to detect an increasing number of mutations, even in the homologous region of the PKD1 gene. Polymerase chain reaction-single-strand conformation polymorphism (PCR-SSCP) or denaturing highperformance liquid chromatography (DHPLC) analyses were performed in the present study to screen mutations from exon 23 to exon 46 in the $P K D 1$ gene and in the entire $P K D 2$ gene. When an abnormal pattern was found in PCRSSCP or DHPLC, the PCR products were directly sequenced. Four mutations were identified in the $P K D 1$ gene: a missense mutation (C47413T causing T3509M in exon 35), a splicing mutation (del $20 \mathrm{bp}$ in $75 \mathrm{bp}$ of intron 43), and two nonsense mutations (C48566A causing C3693X in exon 38, and C51237T causing Q4124X in exon 45). The nonsense mutation Q4124X existed in only two of three affected sib members in family K68. The pattern of the restriction enzyme digest and the haplotype analysis confirmed the presence of a heterozygous mutation in the family. Fifteen single nucleotide polymorphisms were identified in this study. Two of them (C50439A and C51659T) can be used as intragenic polymorphic markers.
\end{abstract}

Key words PKD1 - ADPKD - Mutation - SNP - PCRSSCP $\cdot$ DHPLC

M. Mizoguchi $\cdot$ T. Tamura $\cdot$ A. Yamaki $\cdot$ Y. Shimizu $(\bowtie)$

School of Health Sciences, Kyorin University, 473 Miyashita,

Hachioji, Tokyo 192-8508, Japan

Tel./Fax +81-426-92-0319

e-mail: yshimizu@kyorin-u.ac.jp

M. Mizoguchi

School of Health Sciences, Tokai University, Isehara, Japan

E. Higashihara

School of Medicine, Kyorin University, Tokyo, Japan

\section{Introduction}

Autosomal dominant polycystic kidney disease (ADPKD) is one of the most common hereditary diseases, affecting approximately 1 in 1000 Caucasians. Mutation analysis of the PKD1 gene has been complicated because at least three PKD1-like homologous genes exist on chromosome 16p13.1. Several improved strategies have been developed to detect mutations in the entire PKD1 gene including the duplicated region (Peral et al. 1997; Roelfsema et al. 1997; Watnick et al. 1997; Thomas et al. 1999; Rossetti et al. 2001).

More than 80 mutations, mostly in patients from Western Europe, were identified in the PKD1 gene. These mutations resulted in various alterations of amino acids by substitution, deletion, or insertion of nucleotides (nt). Previous reports suggested that new mutations of the PKD1 gene occur at a significant rate (Peral et al. 1997; Rossetti et al. 2001). Approximately $80 \%$ of the mutations in the 3'PKD1 unique region (exons 34-46) were predicted to truncate the protein, and $37 \%$ of mutations in the 5 'part of the gene were missense mutations (Bogdanova et al. 2000). More mutations were found in exons 15, 23, 25, and 44, but these exons are not considered hot spots for mutations. Because the correlation of $P K D 1$ mutations with genotypes and phenotypes of ADPKD is unclear, it is necessary to accumulate data on $P K D 1$ mutations, especially in different populations.

Although exons 2-22 have been successfully amplified to analyze the duplicated region of the PKD1 gene (Thomas 1999), it has been difficult to amplify the remainder of the region due to the presence of a $2.5-\mathrm{kb}$ polypyrimidine tract in intron 21. Therefore, as a first step, in this study we screened mutations in exons 23-46 of the PKD1 gene and in the entire $P K D 2$ gene from 176 Japanese ADPKD patients. We found four different mutations of the PKD1 gene, one of which was a heterozygous mutation identified in members of the same ADPKD family. 


\section{Subjects and methods}

Patient recruitment. Affected individuals and family members were recruited nationwide through their family physicians at the request of the ADPKD research group, which is supported by the Ministry of Health and Welfare in Japan. Family physicians obtained blood samples from individuals after obtaining their informed consent. Patients were diagnosed at the clinic of their family physician by confirming the presence of more than three cysts in bilateral kidneys by ultrasonography.

$D N A$ and $R N A$ preparation. Genomic DNA was extracted from peripheral lymphocytes by standard phenol/chloroform extraction and isopropanol precipitation. RNA was isolated from established lymphoblast cell lines by using the reagent Trizol LS (GIBCO-BRL, Rockville, MD, USA).

Polymerase chain reaction $(P C R)$ and reverse transcriptase $(R T)-P C R$. Genomic DNA (50ng) was used as a template and amplified by PCR in a $10-\mu l$ reaction volume, containing $10 \mathrm{pmol}$ of each primer and the manufacturer's buffer system in the presence of $10 \%$ dimethylsulfoxide and 0.25 units of rTaq DNA polymerase (Takara, Shiga, Japan) or Pyrobest polymerase (Takara). Eighteen sets of primers for exons 34-46 of the PKD1 gene (nt 44328-52160, accession number L39891) (Peral et al. 1996) and 17 sets of primers for the PKD2 gene were used (Hayashi et al. 1997). PCR was performed according to the method of Peral et al. (1996) with slight modifications. Long-range PCR (Expand Long Template PCR system, Roche, Mannheim, Germany) was performed by using primer set E23F4 (nt 38145-38165) (Koptides et al. 1998) in exon 23 and primer set E34R1 (nt 44403-44422) (Watnick et al. 1997) in exon 34. PCR was performed according to the manufacturer's buffer system 3 method with slight modifications. Amplified DNA products were diluted 1000-10,000 times and were used as templates for nested PCR. Primers were designed to produce 200 $600 \mathrm{bp}$ by dividing them from exon 23 to exon 34 . RT-PCR was performed using random oligonucleotide primers.

PCR-single-strand conformation polymorphism (SSCP). PCR products were diluted 1:10 with solution (95\% formamide, $10 \mathrm{mM} \mathrm{NaOH})$ and heat-denatured at $98^{\circ} \mathrm{C}$ for $5 \mathrm{~min}$. Then they were run on GeneGel 12.5/24 gel (GenePhor System, Pharmacia, Buckinghamshire, UK) or
$6 \%$ polyacrylamide gel. The gels were stained with silver staining kit (Pharmacia) or ethidium bromide.

Denaturing high-performance liquid chromatography (DHPLC). The nesting PCR products were analyzed by WAVE (Transgenomic, Omaha, NE, USA). The manufacturer's solution A $[0.1 \mathrm{M}$ triethylammonium acetate (TEAA)] and solution B (0.1 M TEAA with $25 \%$ acetonitrile) buffer systems were used.

Sequencing. PCR products by Pyrobest polymerase were used as templates in the big-dye dideoxy terminator cycle sequencing method (PRISM 377, ABI).

Restriction enzyme digests. Mae 1 restriction enzyme digests were separated as described earlier.

Haplotype analysis. The haplotype analysis was performed by using four flanking markers (D16S521, 16AC2.5, CW2, and SM7) and two intragenic markers (KG8, I42) for PKD1 and six flanking markers for PKD2 (D4S231, D4S1534, D4S1542, D4S1563, D4S1544, and D4S414) (Harris et al. 1991; Mills et al. 1992; Thompson et al. 1992; Snarey et al. 1994; Viribay et al. 1994; Coto et al. 1995). The 5'end of one of paired primers was labeled with X-rhodamine isothiocyanate (XRITC) and used for PCR. The PCR products were separated by $6 \%$ polyacrylamide gel electrophoresis. Allele patterns were analyzed by FMBIO 100 fluoro-imager (Takara).

\section{Results}

Four mutations in the PKD1 gene were identified from Japanese ADPKD patients (Table 1).

Missense mutation in patients \#250 and \#325

DHPLC analysis showed an abnormal pattern for products in patient \#250 by using primer set I34F/I35R in exons 3435 (Fig. 1a). From direct sequencing analysis, a change of $\mathrm{C}$ to $\mathrm{T}$ at nt 47413 in exon 35 (Fig. 1b) was found. This alteration caused a missense mutation from threonine to methionine at codon 3509, T3509M. The same mutation was found in patient \#325. Because only patients \#250 and \#325

Table 1. $P K D 1$ mutations identified in this study

\begin{tabular}{lllll}
\hline Mutation type & $\begin{array}{l}\text { Family (K) or } \\
\text { patient (\#) }\end{array}$ & Nucleotide change & Amino acid change & Exon/intron \\
\hline Missense & $\# 250, \# 325$ & C47413T & T3509M & Exon 35 \\
Splicing & K97 & del 20 bp & C3693X & Intron 43 \\
Nonsense & \#303, \#312 & C48566A & Qxon 38 \\
& K68 & C51237T & Exon 45 \\
\hline
\end{tabular}


a normal $\# 250$
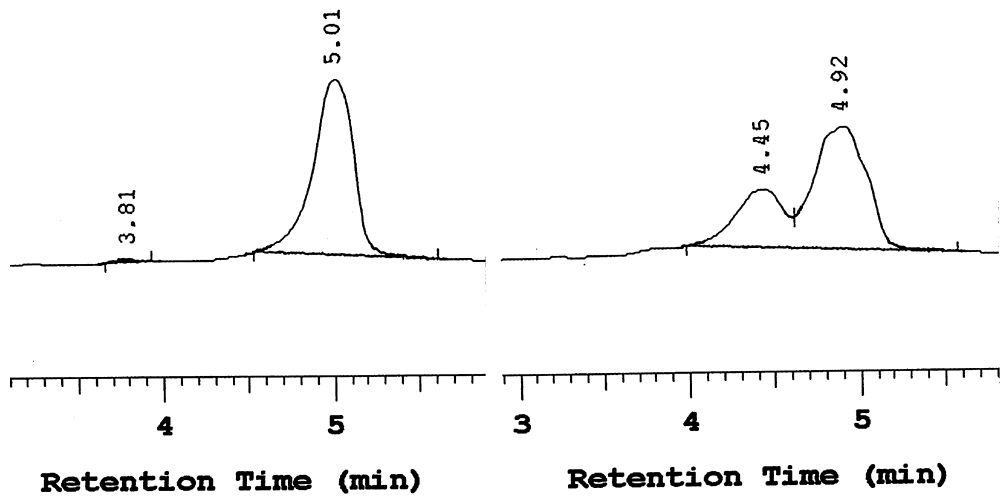

b

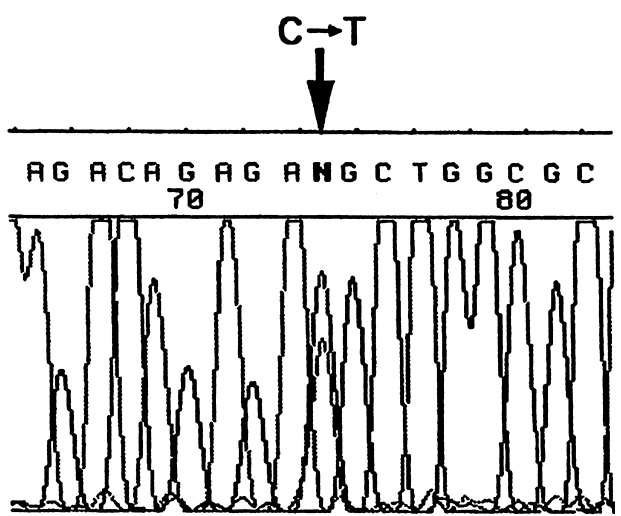

Fig. 1a,b. Missense mutation in patient \#250. a Denaturing high-performance liquid chromatography (DHPLC) patterns in a normal sample (left) and in patient \#250 (right). b The sequence analysis of the polymerase chain reaction product of patient \#250 showed nucleotide change C47413T (arrow), which caused the missense mutation T3509M were available from their families, we were not able to analyze the segregation of this change. However, the same abnormal pattern in DHPLC was not found among the 80 normal samples.

Splicing mutation in family K97

PCR-SSCP analysis using primer set DD in intron 43 (Fig. 2b) showed that affected members I-2, I-3, and II-2 had abnormal bands but unaffected members I-1, I-4, and II-1 did not. From the direct sequencing analysis of all affected members of this family, a deletion of $20 \mathrm{bp}$ was found in a 75-bp length of intron 43 (Fig. 2c). RT-PCR analysis (Fig. 2d) showed that members I-2, I-3, and II-2 had the normal size product (188bp), a 55-bp longer size product (243 bp), and two additional products (593 bp and $403 \mathrm{bp}$ ), whereas members I-1, I-4, and II-1 had only the normal size product (188bp).

Two nonsense mutations in family K68 and in patients \#303 and \#312

In family K68, PCR-SSCP analysis using the primer set $\mathrm{HH}$ in exon 45 showed that affected sisters II-3 and II-5 had an abnormal extra band with two other normal bands, but unaffected sister II-1 and affected sister II-4 did not (Fig. 3b). DHPLC and PCR-SSCP analysis using other primer sets in exons 24-46 showed no other abnormal pattern for any other member. Direct sequencing analysis was performed for all members of this family. Sequencing of II-3 and II-5 (Fig. 3c) revealed a C-to-T substitution $(\mathrm{CAG} \rightarrow \mathrm{TAG})$ at nt 51237, resulting in the nonsense mutation glutamine to stop at codon $4124(\mathrm{Q} 4124 \mathrm{X})$ in exon 45. Digestion of restriction enzyme Mae 1 was performed to confirm the mutation in this family. With this substitution, a new Mae 1 restriction site was created, resulting in two bands (160 bp and 28bp). In members II-3 and II-5, two fragments (188bp and $160 \mathrm{bp}$ ) were detected, whereas only one band (188bp) was detected in members II-1 and II-4 (Fig. 3d). Although the change of amino acid from valine to methionine caused by an alteration of nucleotide at $\mathrm{nt}$ 39034 in exon 25 was found in member II-4, this alteration also existed in member II-1 and in three other unrelated individuals. The haplotype analysis was performed in this family by using six markers each for PKD1 and PKD2. For the PKD1 gene, markers 16AC2.5, CW2, and SM7 were informative. For the PKD2 gene, markers D4S231, D4S1563, and D4S1544 were informative. Only affected members II-3 and II-5 shared the haplotype linked to the PKD1 gene, whereas members II-3 and II-4 shared the haplotype linked to the PKD2 gene (Fig. 3e). The linkage data gave maximum logarithm of differences scores of $-2.71(\theta=0.0178)$ and $-3.14(\theta=0.0148)$, respectively (Lathrop et al. 1985).

One other nonsense mutation was found in patient \#303. DHPLC analysis using the primer set $\mathrm{G} \alpha$ in exons 37-38 showed an abnormal pattern (Fig. 4a). From the direct sequencing analysis, a change of $\mathrm{C}$ to A occurred at nt 48566 in exon 38 (Fig. 4b). This alteration caused the nonsense mutation cysteine to stop at codon 3693 (C3693X). We found the same mutation in patient \#312. Because only patients \#303 and \#312 were available from their families, we were not able to analyze the segregation of this change.

\section{Polymorphism}

During the mutation search, 15 single-nucleotide polymorphisms in the PKD1 gene were found by direct sequencing 
following DHPLC or PCR-SSCP analysis (Table 2). One of these changes resulted in an amino acid substitution, valine to methionine, by converting $\mathrm{G}$ to $\mathrm{A}$ at $\mathrm{nt} 39034$ in exon 25 with a frequency of $3.3 \%$. Although two other polymorphisms, C50439A in exon 43 and C51659T in exon 46, with frequencies of $6.8 \%$ and $8.2 \%$, respectively, caused no amino acid changes, they might be useful as intragenic polymorphic markers.

Table 2. Single nucleotide polymorphisms identified in this study

\begin{tabular}{llll}
\hline $\begin{array}{l}\text { Nucleotide } \\
\text { change }\end{array}$ & $\begin{array}{l}\text { Amino acid } \\
\text { change }\end{array}$ & Site & Frequency (\%) \\
\hline G38976A & Ala2988Ala & Exon 25 & $1 / 66(1.5)$ \\
C38997A & Leu2995Leu & Exon 25 & $1 / 66(1.5)$ \\
G39034A & Val3008Met & Exon 25 & $5 / 149(3.3)$ \\
C47848T & & Intron 36 & $1 / 98(1.0)$ \\
47853delC & & Intron 36 & $1 / 98(1.0)$ \\
C48085T & & Intron 37 & $2 / 96(2.0)$ \\
C48205G & & Intron 37 & $1 / 122(0.8)$ \\
G48509A & Leu3674Leu & Exon 38 & $1 / 97(1.0)$ \\
G49637A & & Intron 40 & $2 / 147(1.4)$ \\
C49833T & & Intron 41 & $1 / 122(0.8)$ \\
T49942G & & Intron 41 & $2 / 124(1.6)$ \\
C49943G & & Intron 41 & $2 / 124(1.6)$ \\
G50075A & Glu3871Glu & Exon 42 & $1 / 122(0.8)$ \\
C50439A & Ala3910Ala & Exon 43 & $9 / 133(6.8)$ \\
C51659T & Asp4234Asp & Exon 46 & $12 / 146(8.2)$ \\
\hline
\end{tabular}

\section{Discussion}

In the present study, we detected four mutations in 176 ADPKD individuals by analyzing exons $23-46$ of the PKD1 gene. From the relatively low rate of mutation detected in this study, we assume that the majority of mutations exist in exons $1-23$ or in the regulator region of the $P K D 1$ gene, as suggested in a previous report (Peral et al. 1997).

A missense mutation (C47413T, causing T3509M in exon 35 ) in patients \#250 and \#325 has not been identified before. In this mutation, threonine has a polar $\mathrm{OH}$ group but methionine does not. Therefore it is possible that this alteration causes the gene to produce a different tertially structured protein. Patient \#250 was a 61-year-old woman who was having hemodialysis treatment. She also had a history of hypertension and has been confirmed as having liver cysts with no symptoms. A sister died from end-stage renal disease (ESRD). Patient \#325 was a 71-year-old woman with no symptoms except hypertension. The phenotypes of these two patients seemed to be no more severe than those of average $P K D 1$ patients.

A splicing mutation in intron 43 has been identified in family K97. From the 20-bp deletion, the size of intron 43 became shortened to $55 \mathrm{bp}$ (Fig. 2c). The same mutation was reported previously in two unrelated families in Europe
Fig. 2a-d. Splicing mutation in family K97. a Pedigree of family K97. Filled symbols are affected individuals and open symbols are unaffected individuals. Males are shown as squares and females as circles. Numbers below symbols represent age in years $(y s)$. b Polymerase chain reactionsingle-strand conformation polymorphism (PCR-SSCP) analysis showed extra bands exist only in affected members II-2, I-3, and II-2. c Diagram of $75 \mathrm{bp}$ of intron 43 shows two possible regions of a 20-bp deletion with a solid line and $a$ dotted line. d Reverse transcriptase-PCR analysis showed 188-bp, 243-bp, 403-bp, and 593bp PCR products when cDNA was used as a template in affected member I-2 and only a 188-bp product in unaffected member I-4
Family $\mathbf{K 9 7}$

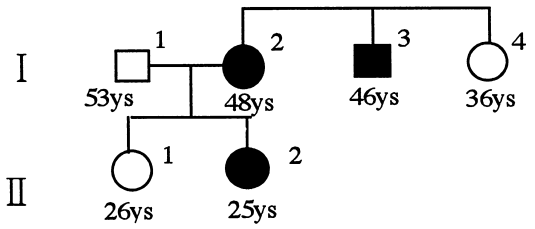

b

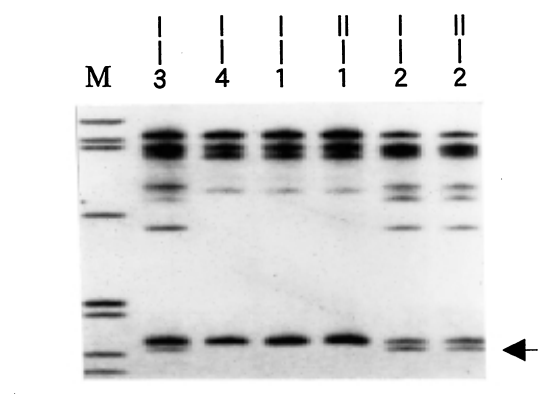

c

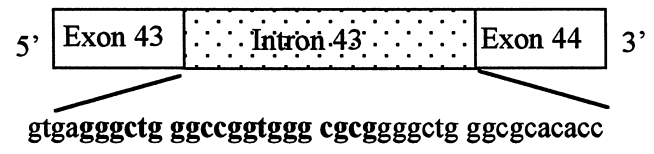

ccagggctgc aagcagacag atttctcgtc cgcag

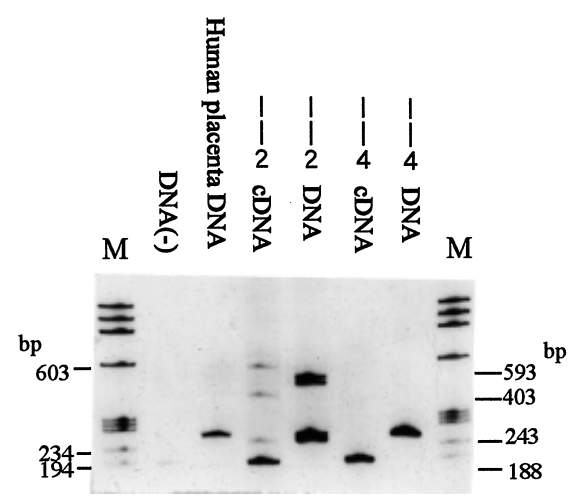


Fig. 3a-e. Nonsense mutation in family K68. a Pedigree of family K68. Affected individuals are indicated by filled symbols. Males are shown as squares and females as circles. Slashes through symbols mean decreased individuals. b PCR-SSCP analysis showed an abnormal extra band (arrow) in members II-3 and II-5 and not in members II-1 and II-4. c The sequence analysis of the PCR product of patient II-3 showed nucleotide change C51237T (arrow), which caused the nonsense mutation Q4124X. d Restriction enzyme Mae 1 digests created the 160-bp band in members II-3, and II-5. e Haplotype analysis in family K68 showed that members II-3 and II-5 shared "4-3-2", members II-3 and II-4 shared "3-2-2" in PKD1 linking markers, and members II-3 and II-4 shared "2-2-2" and "2-2-1" in $P K D 2$ linking markers among the three affected members

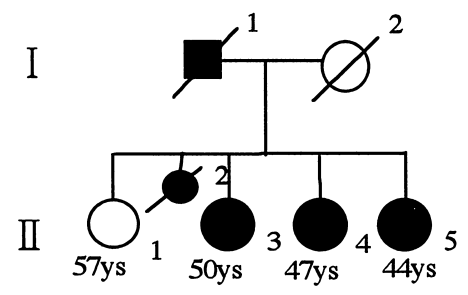

b

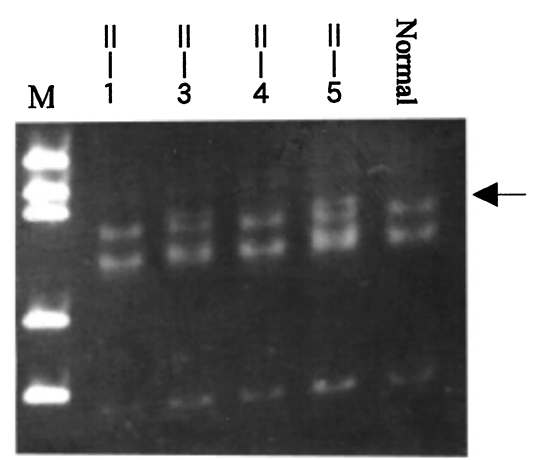

c
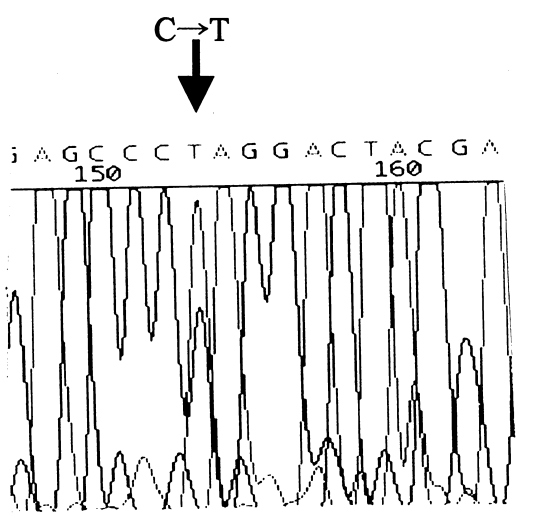

d

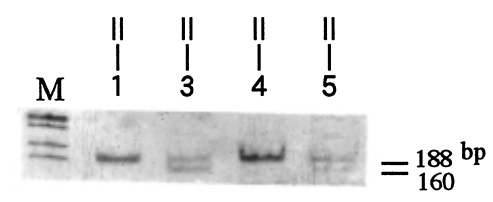

e

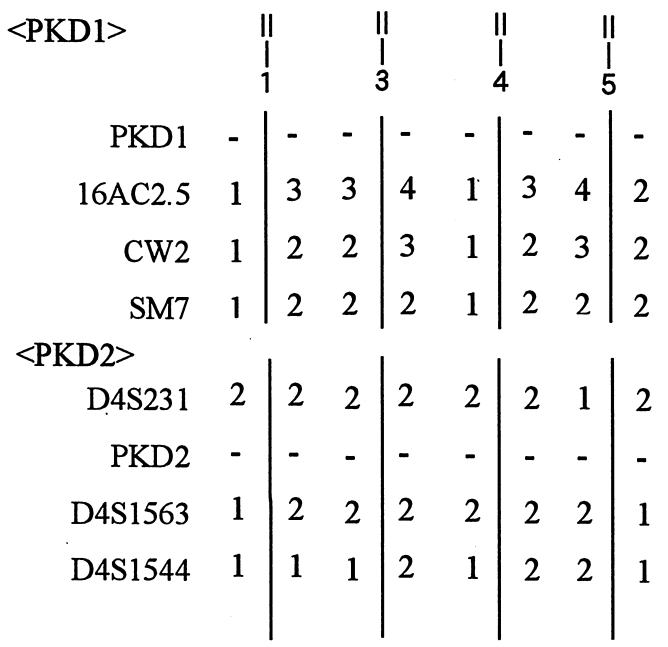

(Peral et al. 1995, 1997). A segment longer than 65 bp is needed to splice the intron correctly (Wieringa et al. 1984). RT-PCR analysis showed that there were three longer bands with a normal sized product (188bp) in patient I-2. The 243-bp product was expected not to be spliced out, and two other larger products (403 bp and 593bp) are not known. This deletion probably was produced by misalign- ment because of the 9-bp repeat (GGGCTGGGC) within the intron. The phenotypes of the affected members in family K97 were shown to be heterogeneous (Fig. 2a). All affected members, I-2, I-3, and II-2, had cysts in bilateral kidneys and hypertension, but only patient I-2 had ESRD and had undergone hemodialysis treatment. Member II-2 had liver cysts, whereas members I-2 and I-3 did not. Two 
$\mathbf{a}$

normal

\#303

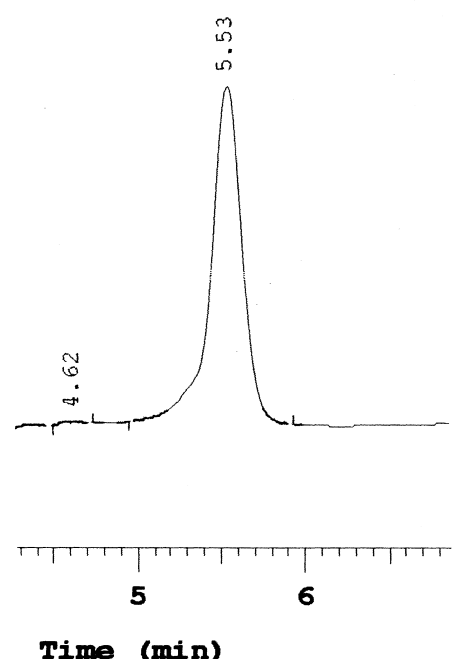

b

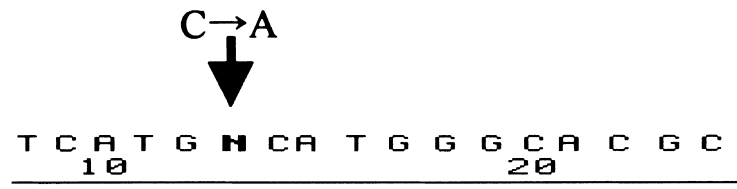

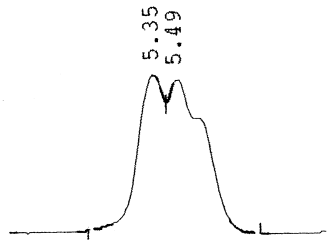

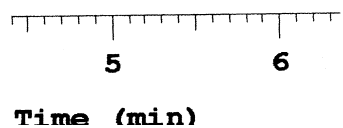

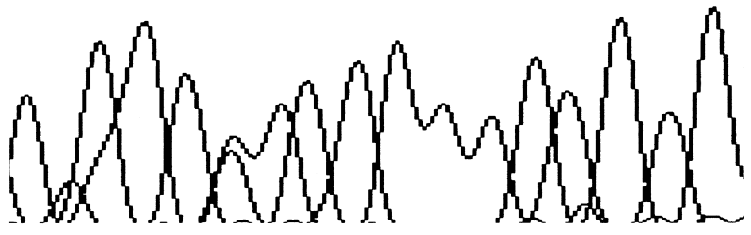

Fig. 4a,b. Nonsense mutation in patient \#303. a DHPLC patterns in a normal sample (left) and in patient \#303 (right) b The sequence analysis showed nucleotide change C48566A (arrow), which caused the nonsense mutation C3693X

previously reported families (Peral et al. 1995) also showed heterogeneous phenotypes. Thus the splicing mutation in intron 43 may not result in severe symptoms.

We report here a nonsense mutation with a heterozygous mutation in family K68 (Fig. 3a). This family includes one unaffected sister, II-1, and three affected sisters, II-3, II-4, and II-5, who were diagnosed by ultrasonography as having cyst formation in bilateral kidneys. All of the affected sisters had hypertension but none of them were treated with hemodialysis. Their father, I-1, and sister, II-2, died of ESRD at the age of 82 years and 52 years, respectively. In our study, two of three affected sisters, II-3, and II-5, had the same mutation (Q4124X) but one sister, II-4, did not. This nonsense mutation was predicted to truncate the protein by 179 amino acids, which is predicted to be the carboxy-terminal domain, which includes the association region with the $P K D 2$ gene product (polycystin2) and signal transduction activities. Digestion by Mae 1 restriction enzyme was performed to confirm that this alteration occurred only in members II-3 and II-5. The result indicated that a new restriction site was created only in the two affected individuals. In member II-4, two possibilities - another unknown mutation or a wrong diagnosis - are suspected. Because patient II-4 was 47 years old and had cyst formations in her bilateral kidneys, we believe the diagnosis was correct. The haplotype analysis in this family showed no link to either the PKD1 or the PKD2 gene. From these results, it is assumed that the mutation in member II-4 happened de novo and that this family has heterozygous mutations of the $P K D 1$ gene because there was no mutation in all exons of the $P K D 2$ gene. Recently it was reported that ADPKD was caused by mutation in the $P K D 1$ gene and/or the $P K D 2$ gene in a large family whose haplotype analysis showed linkage to neither $P K D 1$ nor
$P K D 2$, although the $P K D 1$ gene mutation was not identified (Pei et al. 2001).

Patient \#303 was a 44-year-old woman with another nonsense mutation. She had no symptoms except for liver cysts. Her family members showed various phenotypes. Her father inherited ADPKD from his mother, and 3 of his 11 siblings died from ESRD. She had one sister who also died from ESRD and other three siblings who were healthy. Patient \#312 has the same mutation as patient \#303. She was 46 years old and inherited ADPKD from her father. Although this mutation was predicted to truncate the protein by 610 amino acids, including six transmembrane domains and the cytoplasmic tail, the phenotype of both patients was not severe.

Because no conclusion can be drawn about the correlation between the types of mutations and phenotypes, more data must be accumulated on the mutations of the PKD1 and $P K D 2$ genes. Additional mutation analysis will lead to a useful DNA-based diagnostic test.

Acknowledgments We thank the families and their family doctors for blood samples. This study was supported in part by a Grant-in-Aid for Scientific Research from the Ministry of Education of Japan, a Project Research Grant of Kyorin University, and a program grant from the Ministry of Health and Welfare of Japan.

\section{References}

Bogdanova N, McCluskey M, Sikmann K, Markoff A, Todorov V, Dimitrakov D, Schiavello T, Thomas M, Kalaydjieva L, Dworniczak B, Horst J (2000) Screening the 3'region of polycystic kidney disease 1 (PKD1) gene in 41 Bulgarian and Australian kindreds reveals a prevalence of protein truncating mutations. Hum Mutat 16:166174 
Coto E, de Castro S, Aguado S, Alvarez J, Arias M, Menendez MJ, Lopez-Larrea C (1995) DNA microsatellite analysis of families with autosomal dominant polycystic kidney disease types 1 and 2: evaluation of clinical heterogeneity between both forms of the disease. $\mathbf{J}$ Med Genet 32:442-445

Harris PC, Thomas S, Ratcliffe PJ, Breuning MH, Coto E, LopezLarrea C (1991) Rapid genetic analysis of families with polycystic kidney disease 1 by means of a microsatellite marker. Lancet 338:1484-1487

Hayashi T, Mochizuki T, Reynolds DM, Wu G, Cai Y, Somlo S (1997) Characterization of the exon structure of the polycystic kidney disease 2 gene (PKD2). Genomics 44:131-136

Koptides M, Constantinides R, Kyriakides G, Hadjigavriel M, Patsalis PC, Pierides A, Deltas CC (1998) Loss of heterozygosity in polycystic kidney disease with a missense mutation in the repeated region of PKD1. Hum Genet 103:709-717

Lathrop GM, Lalouel JM, Julier C, Ott J (1985) Multilocus linkage analysis in humans: detection of linkage and estimation. Am Hum Genet 37:402-498

Mills KA, Buetow KH, Xu Y, Weber JL, Altherr MR, Wasmuth JJ, Murray JC (1992) Genetic and physical maps of human chromosome 4 based on dinucleotide repeats. Genomics 14:209-219

Pei Y, Paterson AD, Wang KR, He N, Hefferton D, Watnick T, Germino GG, Parferey P, Somlo S, St. George-Hyslop (2001) Bilineal disease and trans-heterozygotes in autosomal dominant polycystic kidney disease. Am J Hum Genet 68:355-363

Peral B, Gamble V, San Millan JL, Strong C, Sloane-Stanley J, Moreno F, Harris PC (1995) Splicing mutations of the polycystic kidney disease 1 (PKD1) gene induced by intronic deletion. Hum Mol Genet 4:569-574

Peral B, Ong ACM, Gamble V, Ward CJ, Strong C, Harris PC (1996) Screening the 3 'region of the polycystic kidney disease 1 (PKD1) gene reveals six novel mutations. Am J Hum Genet 58:86-96

Peral B, Gamble V, Strong C, Ong ACM, Sloane-Stanley J, Zerres K, Winearls CG, Harris PC (1997) Identification of mutations in the duplicated region of the polycystic kidney disease 1 gene (PKD1) by a novel approach. Am J Hum Genet 60:1399-1410

Roelfsema JH, Spruit L, Saris JJ, Chang P, Pirson Y, van Omen G-JB, Peters DJM, Breuning MH (1997) Mutation detection in the repeated part of the PKD1 gene. Am J Hum Genet 61:1044-1052

Rossetti S, Strmecki L, Gamble V, Burton S, Sneddon V, Peral B, Roy S, Bckkaloglu A, Komel R, Winearls CG (2001) Mutation analysis of the entire PKD1 gene: genetic and diagnostic implications. Am J Hum Genet 68:46-63

Snarey A, Thomas S, Schneider MC, Pound SE, Barton N, Wright AF, Somlo S, Germino GG, Harris PC, Reeders ST, Frischauf AM (1994) Linkage disequilibrium in the region of the autosomal dominant polycystic kidney disease gene (PKD1). Am J Hum Genet 55:365-371

Thomas R, McConnell R, Whittacker J, Kirkpatrick P, Bradley J, Sandford R (1999) Identification of mutations in the repeated part of the autosomal dominant polycystic kidney disease type 1 gene, PKD1, by long-range PCR. Am J Hum Genet 65:39-49

Thompson AD, Shen Y, Holman K, Sutherland GR, Callen DF, Richards RI (1992) Isolation and characterization of (AC) microsatellite genetic markers from human chromosome 16. Genomics 13:402-408

Viribay M, Ferreira R, Peral B, Bello D, Ward CJ, Davalos J, Valle C, Harris PC, Castillo DM, Moreno F, Millan JS (1994) Genetic analysis of Cuban autosomal dominant polycystic kidney disease kindreds using RFLPs and microsatellite polymorphisms linked to the PKD1 locus. Hum Genet 94:432-436

Watnick TJ, Piontek KB, Cordal TM, Weber H, Gandolph MA, Qian F, Lens XM, Neumann HPH, Germino GG (1997) An unusual pattern of mutation in the duplicated portion of $P K D 1$ is revealed by use of a novel strategy for mutation detection. Hum Mol Genet 6:1473-1481

Wieringa B, Hofer E, Weissmann C (1984) A minimal intron length but no specific internal sequence is required for splicing the large rabbit $\beta$-globin intron. Cell 37:915-925 